\title{
Desafíos en el diseño e implementación del Programa Integral de la Cuenca del Arroyo San Antonio de la Universidad de la República
}

\section{Maximiliano Piedracueva \\ maxipc85@gmail.com}

Leticia Benelli

lebenro@gmail.com

Lucía Holly

luciaholly1@gmail.com

Victoria Bandera

victoria.b90@gmail.com
Docentes del Centro Universitario Regional Litoral Norte Salto de la Universidad de la República, Uruguay.
Integración de la docencia y la extensión /

Desafíos de gestión

RECEPCIÓN: 24/06/16

ACEPTACIÓN FINAL: 07/09/16

\section{Resumen}

En el año 2015, el Centro Universitario Salto de la Universidad de la República (UdelaR) comienza el diseño del Programa Integral de la Cuenca del Arroyo San Antonio (PICASA). Dicho programa surge como política institucional de extensión universitaria con el objetivo de coordinar y articular las actividades de enseñanza, extensión e investigación que se realizan en dicho espacio. El presente artículo da cuenta del proceso de diseño e implementación del PICASA en un contexto universitario particular dadas las características del Centro Universitario y las transformaciones políticas e institucionales de la extensión universitaria en UdelaR. Reflexiona sobre este dispositivo institucional denominado Programa Integral y sobre los desafíos metodológicos, conceptuales, institucionales y de gestión que se presentan en su implementación.

Palabras-clave

- Integralidad de funciones

- Política de extensión universitaria

- Programas integrales

\section{Resumo}

Em 2015 o Centro Universitário Salto da Universidad de la República começa a idear o Programa Integral de la Cuenca del Arroyo San Antonio (PICASA). Este Programa surge como política institucional de extensão universitária com o objetivo de coordenar e articular as atividades de ensino, extensão e pesquisa, que se realizam nesse espaço. Este artigo informa sobre o processo de criação e da implementação do PICASA num contexto universitário particular pelas características do Centro Universitário e pelas transformações políticas e institucionais da extensão universitária na Universidade da República. Reflete sobre o dispositivo institucional chamado Programa Integral e sobre os desafios metodológicos, conceituais, institucionais e de gestão que se apresentam na sua implementação.

Palavras-chave

- Integralidade de funções

- Política de extensão universitária

- Programas integrais
一

oste artículo

Piedracueva, M.; Benelli, L.; Holly, L. y Bandera, V. (2016).

Desafíos en el diseño e implementación del Programa Integral de la Cuenca del Arroyo San Antonio de la Universidad de la República. En Revista +E versión digital, (6), pp. 146-153. Santa Fe, Argentina: Ediciones UNL. 


\section{Introducción ${ }^{1}$}

La Universidad de la República (UdelaR) adscribe al Modelo Latinoamericano de Universidad y cuenta con tres funciones esenciales que hacen a la vida y formación universitaria: enseñanza, extensión e investigación. En los últimos años, UdelaR se encaminó en un proceso que dio a llamarse la $2^{\text {a }}$ Reforma Universitaria, la cual contemplaba, dentro de otras, la intención de promover la extensión universitaria como práctica docente y estudiantil. A esos fines, el Servicio Central de Extensión se encaminó en la discusión, reflexión y conceptualización de las prácticas integrales como dispositivos académicos que permitan la inclusión de la extensión en el conjunto de actividades realizadas por los/as universitarios/as. Las prácticas integrales pueden entenderse a partir de los siguientes ejes temático o componentes (Tomasino y otros, 2010): a) la integración de los procesos de enseñanza y creación de conocimiento a experiencias de extensión;

b) la perspectiva interdisciplinaria tanto en el nivel epistemológico vinculado a la enseñanza (tratamiento de los contenidos) y a la creación de conocimiento (construcción del objeto de investigación), como en el nivel de la intervención (construcción y abordaje de los problemas, conformación de los equipos); c) la intencionalidad transformadora de las intervenciones, concibiendo a los actores sociales como sujetos protagonistas de dichas transformaciones y no como objeto de intervenciones universitarias (participación comunitaria, diálogo de saberes y ética de la autonomía);

d) la concepción integral de los procesos de enseñanza y aprendizaje, tanto en el tratamiento de los contenidos como en las metodologías (ecología de saberes, enseñanza activa, aprendizaje por problemas);

e) Enfoque territorial e intersectorialidad en el abordaje de las intervenciones.

El concepto de prácticas integrales se encuentra directamente relacionado con el de extensión universitaria. Debe señalarse que en UdelaR no existe un consenso sobre la noción de extensión universitaria y por lo tanto es prácticamente imposible, quizás innecesario, intentar brindar una definición conceptual. Si bien en los últimos años docentes y estudiantes se han abocado a discutir y reflexionar sobre esta función, existen elementos de tipo político y teórico-metodológico que asientan una base de inconmensurabilidad en la discusión. En este artículo se adscribe al concepto aprobado por UdelaR en el año 2009 y que aborda a la extensión universitaria desde cuatro grandes dimensiones (UdelaR CCEA, 2008; UdelaR CSEAM, 2011). Una primera dimensión de la extensión universitaria es la pedagógica, la cual refiere al rol educativo que tienen las prácticas de extensión. La resolución de UdelaR sobre este punto sostiene que la extensión es un "proceso educativo transformador donde no hay roles estereotipados de educador y educando, todos pueden aprender y enseñar" (CDC, 2009).

Esta afirmación contiene dos grandes conceptos, uno educativo y uno pedagógico. El primero de ellos refiere a la importancia de las prácticas de extensión en el proceso de formación de estudiantes universitarios y a que estas prácticas son un aporte sustancial a la formación específica disciplinar pues ofrecen un formato a la "intemperie del aula", al decir de Tommasino y Rodríguez (2010). Se trata de una expresión que permite describir el trabajo en realidades concretas e intenta aprender, enseñar y resolver algunos de los problemas con la población involucrada. Es un trabajo a nivel comunitario o social donde aparecen elementos novedosos debido a que es una realidad que se conoce siempre parcial y provisoriamente. De esta forma, la comprensión cabal de la situación se ve facilitada cuando se cuenta con un equipo interdisciplinario. Aparecen el actor social y el medio como una propuesta enseñante más allá del vínculo estudiante/docente, y esto desestructura la relación de poder que normalmente se establece en el acto educativo.

El segundo concepto apunta a cómo se deben implementar dichas prácticas con un objetivo educativo, y sobre ello establece que se debe romper con la estructura jerárquica entre docentes y estudiantes. Tal concepción tiene bases epistemológicas no conservadoras en cuanto a la construcción de conocimiento ya que asume que todas las personas pueden, en un mismo acto pedagógico, aprender y enseñar (Piedracueva, 2016). La dimensión pedagógica de la extensión no solo rompe con la estructura tradicional de la formación universitaria entre docentes y estudiantes sino que la modifica también en cuanto al vínculo entre estudiantes. Desde esta concepción se establece la posibilidad y necesidad de que estudiantes con distintos niveles de avance en la carrera puedan participar y compartir espacios de formación curriculares. 
Una segunda dimensión de la extensión es la ética, que establece la horizontalidad y el respeto con el cual los/as universitarios/ as deben acercarse a las realidades concretas de intervención. ${ }^{2}$ En el momento en el que los/as universitarios/as emprenden un proceso de intervención con determinada población deben ser conscientes, por una parte, del conocimiento disciplinar específico con el que cuentan, y por otra parte, de las metodologías adecuadas que deben ser empleadas para establecer un proceso dialógico entre los distintos saberes. La extensión culturalista en la cual quienes van desde la universidad deben "iluminar" a los "no cultos" es, desde este punto de vista, una falta ética. También lo es que quienes provengan desde la universidad nieguen su calidad de universitarios/as y desconozcan sus conocimientos específicos disciplinares.

Una tercera dimensión es la metodológica, y en este sentido la extensión universitaria no debe llevarse a cabo con cualquier estrategia. Las prácticas de extensión deben implementarse, para dar sentido a las anteriores dimensiones, a través de herramientas que faciliten la participación. El proceso dialógico de saberes entre quienes se encuentran involucrados/as en dichas prácticas (docentes, estudiantes, no universitarios) requiere de la implementación de diseños metodológicos rigurosos que fomenten la participación de todos los actores involucrados. Teniendo en cuenta lo anterior, es importante que la extensión universitaria integre diseños y herramientas que faciliten la participación activa de la población y los estudiantes en la problematización así como en las estrategias de trabajo sobre los problemas identificados. La resolución del CDC (2009) establece que la extensión universitaria "promueve formas asociativas y grupales de trabajo que aporten a superar problemáticas significativas a nivel social".

El carácter interdisciplinario de la extensión es, en realidad, un deseo más que una práctica. Asumiendo que la realidad no es disciplinar (no es económica, arquitectónica, agronómica, social, etc.) sino que es un entramado de relaciones en las que intervienen diversos factores, es lógico pensar que no puede ser abordada disciplinariamente. Sin embargo la puesta en práctica de un abordaje interdisciplinar es compleja dadas las distintas formaciones. La interdisciplina es una práctica metodológica y por lo tanto debe pensarse en un cómo hacer un objeto de intervención entre diferentes disciplinas. En los hechos, las prácticas universitarias han logrado establecer intervenciones multidisciplinares, esto es, que distintas personas con diferentes formaciones disciplinares intervienen sobre un mismo grupo o una misma comunidad, mas no sobre un mismo objeto. Debe señalarse el riesgo que se corre en la búsqueda de la interdisciplina. Que personas con diversas formaciones disciplinares participen en una actividad de extensión no asegura la multidisciplina, y menos aún la interdisciplina. Puede ocurrir que se trate de un voluntariado, es decir, de la realización de prácticas de intervención sin vínculo disciplinar. Si no se construye intelectualmente un objeto de intervención existen posibilidades de que personas de diversas disciplinas intervengan sobre un objeto construido desde una de ellas.

Una última dimensión de la extensión es la política. Aquí se define a la extensión universitaria como crítica en contraposición a la culturalista y la difusionista. El objeto de intervención de la extensión son los problemas sociales y éstos deben partir de una lectura crítica y colectiva de la realidad. La extensión en su dimensión política niega la intervención desde una lógica de adiestramiento y de soluciones mágicas, refuerza la idea de la construcción social de la realidad y de la existencia de conflictos latentes en los procesos sociales. La política universitaria debe perseguir principios de igualdad y equidad y por ende propender al trabajo con aquellos sectores de la sociedad que se encuentran en peores condiciones de ejercer sus derechos. Entendiendo que las prácticas de extensión forman parte de la formación universitaria, parte del aprendizaje debe ser identificar quiénes pueden necesitar los servicios universitarios. A cada quien según sus necesidades y cada cual según sus posibilidades.

\section{El Programa Integral de la Cuenca del Arroyo San Antonio} El Programa Integral Cuenca del Arroyo San Antonio (PICASA) es un emprendimiento del Centro Universitario Salto de la UdelaR que busca implementar prácticas de enseñanza, extensión e investigación de manera coordinada e integrada, con participación de la comunidad en el territorio delimitado por la cuenca del Arroyo San Antonio en el departamento de Salto, Uruguay. El área determinada es mayoritariamente rural y cuenta con algunos núcleos poblacionales pequeños. La población total del área es
2) En el equipo se ha planteado la discusión sobre la utilización del término intervención, entendiéndose como un proceso colectivo que tanto en la etapa del pensar como en la del hacer integra actores universitarios y sociales en la búsqueda de construir la demanda en forma conjunta y programar propuestas de acción participativas.
Aquí este término hace referencia a distintos abordajes colectivos en terreno. Busca diferenciarse de la idea de intervención que responde al modelo médico cientificista positivista, que si se extrapola a los procesos subjetivos de construcción humana, tiende a deconstruir. Implica abrir, extirpar, sacar lo que está mal, donde el técnico se posiciona desde un supuesto saber para detectar, digitar, inmiscuirse con otros a los cuales considera "fallados" y reparar esa falla. Tal como lo señala Montero (2012), pueden encontrarse más de 39 verbos del idioma español relacionados con el concepto de intervención, desde accionar hasta invadir. La propuesta del PICASA respecto de la intervención sugiere que ésta sea entendida como praxis; sin embargo, en el documento se hace referencia a la intervención sin un vínculo directo con alguna postura teórica dado que entre las diferentes disciplinas que participan en el Programa no hay, aún, un acuerdo sobre el concepto y sus implicancias. 
de 6131 personas (INE, 2011), de las cuales un $57 \%$ reside en el medio rural disperso y el restante $43 \%$ en centros poblados. Aproximadamente un $20 \%$ de las personas reside en hogares que cuentan con al menos tres Necesidades Básicas Insatisfechas. De estas personas, un $31 \%$ se declaran inactivas y un $28 \%$ son niños/as menores de 12 años y residen en aproximadamente unas 300 viviendas, las cuales se ubican principalmente en el medio rural disperso (46\%) y en la localidad de San Antonio (24,5\%), en donde se ha definido centrar las acciones del Programa en su primera etapa (datos elaborados por el equipo de PICASA sobre la base del Censo INE 2011).

Los objetivos del Programa apuntan a contribuir a la creación y al fortalecimiento de un espacio académico formal e institucionalizado de carácter integral e interdisciplinario que permita la intervención coordinada y articulada de los distintos servicios universitarios a través de la realización de actividades de enseñanza, extensión e investigación. Para ello se busca desarrollar alternativas conjuntas entre actores universitarios y actores sociales que contribuyan al logro de una mejor calidad de vida de la comunidad involucrada, apostando a la construcción de un espacio de formación y aprendizaje con base en problemas emergentes de la realidad en la que se encuentran e interactúan los distintos saberes y disciplinas; además se apunta a la construcción de un espacio de trabajo y diálogo entre el Centro Universitario Salto y la sociedad civil organizada de la Cuenca del Arroyo San Antonio. Los principios orientadores que guían el desarrollo de PICASA son: la relación sociedad-universidad sustentada en la participación y el diálogo de saberes, la articulación de funciones y la concepción interdisciplinaria, la búsqueda colectiva y participativa de soluciones a problemas significativos, el trabajo en red y el enfoque territorial, los procesos de enseñanza y aprendizaje inclusivos y promotores de una ética de la autonomía, la participación y el diálogo, la gestión participativa y la comunicación, seguimiento, evaluación y sistematización participativos.

Actualmente, docentes y estudiantes de diversas disciplinas se encuentran en plena formulación e implementación de prácticas integrales que articulan principalmente las funciones de enseñanza y extensión; los saberes académicos que confluyen en esta experiencia provienen del área de las Ciencias Sociales, Enfermería, Psicología, Arquitectura, Agronomía y Estudios turísticos (Humanidades).

\section{Desafíos conceptuales y metodológicos}

Las prácticas integrales, como se ha dicho, tienen un fin educativo y transformador. Esto es que las actividades de enseñanza, de extensión y de investigación no sólo confluyen en un lugar determinado sino que deben necesariamente coordinar y articular sus objetivos, sus métodos y sus técnicas. La condición indisciplinada de la realidad requiere para su intervención la conjunción de diversos saberes, métodos y herramientas. Si bien esta afirmación suele ser compartida la puesta en práctica de tal postulado conforma un desafío sustancial en la integralidad de funciones. En el PICASA participan disciplinas de diversas áreas académicas: científico tecnológica, salud, social y artística. Esta variedad al tiempo que enriquece, complejiza el proceso de implementación del programa, dado que se presenta con experiencias y conceptualizaciones de extensión diferentes y por otra parte no todas las carreras cuentan con componentes que apunten a la formación para la intervención, Por tanto los/ as estudiantes y docentes que participan en el programa parten desde distintos lugares. Por otra parte existen carreras que cuentan con formación en intervención pero sin compartir modelos teóricos y conceptuales comunes, y en algunos casos suelen ser inconmensurables.

Esta primera dificultad se intenta subsanar con la realización de un seminario de extensión en el cual participan estudiantes de las diversas carreras como paso previo al trabajo de campo en la localidad de San Antonio. Se busca construir un universo conceptual y epistemológico, acordado, desde una perspectiva de extensión universitaria crítica, aportando algunos conocimientos básicos sobre herramientas que propicien la participación y horizontalidad en el vínculo entre los actores involucrados.

La puesta en práctica de este seminario ha sido un gran desafío y a la vez una potencialidad, ya que posibilita la comprensión de los fenómenos de manera colaborativa y apunta a disminuir la parálisis epistemológica generada cuando la comprensión se realiza desde una sola perspectiva, constituyéndose así en un componente esencial para el equipo del programa, dado que se han evidenciado diferencias epistemológicas sustanciales en la formación académica de las carreras que participan. Estas formaciones enfrentan de manera constante corrientes epistemológicas del positivismo lógico, del falsacionismo y de la pluralidad epistemológica propuesta desde el concepto de extensión. La propuesta del PICASA es que el conocimiento se construye a partir de relaciones sociales. En estas relaciones intervienen personas, contextos determinados, objetos materiales, elementos simbólicos y distintos saberes, lo que da cuenta de que cualquier dato construido en un contexto determinado, por un grupo de personas determinadas, con un cúmulo de saberes determinados, no debe ser necesariamente pasible de su reconstrucción con otras personas, otros contextos y otros saberes.

Junto con esta dificultad existen algunos desafíos en cuanto a la funcionalidad del dato y de la información en un proceso de intervención. EI PICASA establece que el relevamiento de datos se da en un marco de diagnóstico para transformar. En este sentido, las distintas formaciones universitarias no cuentan con una corriente común que permita generar acuerdos y consensos y se presenta siempre la discusión de "relevar para qué y cómo." De este modo se intenta realizar en conjunto la construcción epistemológica en el marco del Programa mediante la generación 
de acuerdos sobre cómo concebir esos datos y su construcción en todas sus dimensiones.

En la generación de estos acuerdos es importante romper con la rigidez del ego epistemológico durante todo el proceso, en la comprensión del fenómeno pero sobre todo en la construcción humana del equipo. Se considera sustancial en estos procesos profundizar en el trabajo interno teniendo en cuenta la contradicción que es propia del ser humano como una de las grandes dificultades en la construcción con otros, lo que genera la necesidad de poner en juego los egos.

Como expresa Carrizo:

"los valores forjados en la experiencia interdisciplinaria contribuyen a fortalecer el espíritu de participación, responsabilidad, integración y mutua comprensión. Por su propia esencia relacionada con el conocimiento y con su aplicación al mundo real, la interdisciplinariedad se proyecta en una perspectiva ética de la investigación y la educación. Educar hoy es también educar en valores, quizás no tanto a través de lecciones morales, sino con una profunda y sistemática reflexión sobre los quehaceres y los modos de pensar". (2010:13)

Con referencia a lo anterior, el equipo del Programa manifiesta un particular interés ya que considera que hay valores que transversalizan el quehacer en el marco del mismo, que permiten desarrollar y sostener este proceso de trabajo interdisciplinario. La construcción humana del equipo cuenta con diversas aristas. Por una parte, se ponen en juego aquellos componentes que llegan junto con las personas (valores, formación, experiencia) y los que se construyen en y con el colectivo (nuevos valores, nuevos conocimientos, nuevas experiencias, roles, funciones, etc.). Un desafío importante para el equipo de trabajo es conjugar los diversos componentes de cada una de las personas que se integran a él desde diversos lugares, intereses, tiempos, entre otras características. El rol y la función que cada persona cumple se construyen en la práctica colectiva y ese es un aspecto desafiante para el Programa debido a la multiplicidad de personas, a la cuantía, por ejemplo, de estudiantes universitarios que participan en el mismo y a la dificultad que ello conlleva en la distribución de roles y en la diversidad que se presenta al momento de asumir responsabilidades y protagonismos.

La puesta en marcha del Programa ha convivido con la antigua, clásica y no por ello desactualizada tensión entre teoría y práctica. Desde las reflexiones y discusiones dadas por idealistas, realistas y materialistas en el campo de la filosofía, pasando por los aportes de Marx en su Tesis sobre Feuerbach (1966), la teoría crítica (Adorno y Horkheimer, 1998), hasta las últimas discusiones latinoamericanas de la mano de Paulo Freire $(1967,1971)$ y Fals Borda (1981), se ha mantenido una constante tensión entre aquello que se conoce y aquello que se hace, y desde la teoría crítica se ha incorporado aquello que se transforma. Por praxis se entiende:

"un modo de producir conocimiento en la acción reflexionada, conducente a la transformación de circunstancias sociales con incorporación de actores sociales comprometidos con esa transformación" (Moreno, 2012:71).

Esta acepción es trabajada en el marco del Seminario de Extensión y forma parte del seguimiento permanente del equipo del Programa. No obstante lo anterior, y aunque existan algunos acuerdos respecto de los postulados centrales de la extensión crítica y de su concepto de praxis, el equipo encuentra una distancia entre el nivel discursivo y el nivel de lo práctico. Esa brecha entre los postulados de la praxis y la "puesta en práctica de la misma" responde a diversos aspectos y características que van desde lo teóricopedagógico hasta lo institucional-administrativo. Los desafíos a los que se enfrenta el equipo son mantener una permanente vigilancia sobre los diferentes abordajes que se realizan en el marco del Programa y el aumento-reducción de la brecha entre lo discursivo y lo práctico.

En este marco, avanzar hacia la consolidación de espacios de encuentro y articulación tanto entre los campos de conocimiento como entre los distintos territorios en donde se construyen los aprendizajes implica mantener una actitud de apertura a nuevas realidades. En este sentido:

"el concepto de frontera sugiere algo muy subversivo y desestabilizador. Significa moverse en círculos de incertidumbre, significa cruzar a diferentes esferas culturales, significa reconocer la naturaleza múltiple de nuestras propias identidades. Significa entender y cuestionar y no tanto asumir una especie de seguridad dentro de las disciplinas académicas. (...) Nos tenemos que introducir en otras esferas donde asumamos la especificidad de diferentes contextos, geografías, lenguajes diferentes, de la alteridad y reconozcamos esa alteridad en nosotros mismos". (Giroux, 1999, en Bordoli, 2010)

Habitar y resignificar colectivamente las fronteras entre los saberes son otros de los desafíos por los que transita el Programa, que apuesta a la transformación de costumbres y límites arraigados institucionalmente.

\section{Desafíos institucionales y de gestión}

El Centro Universitario Salto cuenta con una ventaja comparativa que es la convivencia de diversos servicios universitarios en un mismo espacio físico. Esta característica es un insumo central al momento de transformar en prácticas las dimensiones de la extensión. La posibilidad de llevar adelante prácticas 
los desafíos a los que se enfrenta el equipo son mantener una permanente vigilancia sobre los diferentes abordajes que se realizan en el marco del Programa y el aumento-reducción de la brecha entre lo discursivo y lo práctico

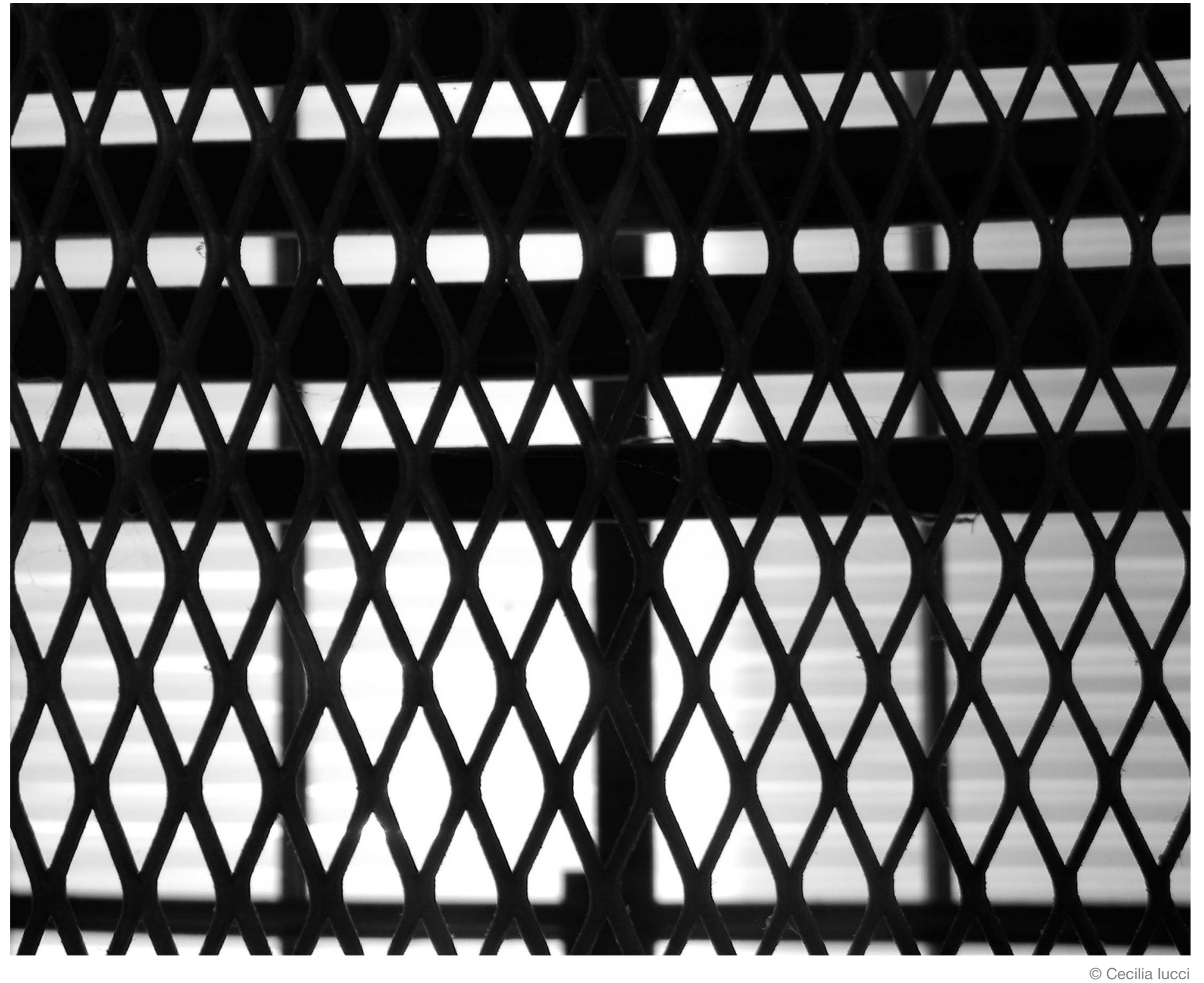


interdisciplinarias se fortalece en la región debido a la variedad de servicios existentes y a la convivencia de los mismos. Como ejemplos concretos deben citarse la ventaja de que los órganos de cogobierno tengan una conformación multidisciplinaria y que las Unidades de Extensión cuenten con la misma característica.

En cuanto a la dimensión pedagógica de las prácticas de extensión, debe señalarse como fortaleza la flexibilidad de varias de las carreras impartidas en los centros universitarios (por ejemplo, los $\mathrm{CIO}),{ }^{3}$ que permiten que los estudiantes compartan espacios académicos con otros estudiantes de diversas formaciones disciplinarias. La puesta en práctica del PICASA posibilita que estudiantes y docentes de diferentes disciplinas compartan no solo espacios áulicos sino también espacios de trabajo de campo, donde se produce un diálogo de disciplinas y de saberes. Se destaca asimismo la tendencia a la conformación de un espacio académico integrado por docentes de diferentes disciplinas que se materialice en un modelo de docencia universitaria en el cual se pueda dar un encuentro epistemológico y el diseño de proyectos de extensión para fortalecer el Programa.

Y no obstante los servicios universitarios muestran una gran heterogeneidad en sus diseños institucionales, a nivel local esto se traduce en diferentes grados de decisión por parte de los servicios universitarios de la sede sobre la incorporación de nuevos espacios o prácticas a las currículas, lo que se presenta en ocasiones como una barrera para el desarrollo de propuestas pedagógicas con orientación a la extensión crítica. En ese contexto, algunos de los servicios mantienen altos grados de dependencia con los niveles centrales de sus servicios de referencia, lo que les impide generar cambios y modificaciones en las estructuras académicas y en los planes de estudio. Otro reto en el ámbito de lo pedagógico se visualiza en la evaluación estudiantil y la valoración de contenidos académicos que se jerarquizan en el proceso de enseñanza. En la heterogeneidad de enfoques que presenta cada facultad y cada curso, las evaluaciones no son un problema en sí, sino que el desafío se centra en innovar en las formas y modalidades de evaluación teniendo en cuenta los marcos institucionales, sobre la base de un universo epistemológico acordado siguiendo la lógica de los planteado en este artículo sobre los desafíos conceptuales. Por otra parte, la dimensión metodológica de la extensión cuenta, como se señaló, con una clara potencialidad referida al diseño e implementación de prácticas multidisciplinares. Junto con ello, al tener el Centro Universitario una zona geográfica concreta de referencia, es factible establecer procesos de acumulación de conocimiento de fácil acceso (tanto a nivel de congresos como de intercambios informales) y, al menos en lo abstracto, emprender procesos de participación de largo aliento en conjunto con la comunidad. Las dificultades residen, al igual que en lo pedagógico, en la dependencia de algunos servicios con el nivel Central. En tanto, igualmente es un desafío al cual se enfrenta el Programa el manejo ético y cuidadoso del trabajo de la Universidad en el medio. La circunscripción geográfica, así como es ventaja es una dificultad. El espacio geográfico es limitado y acotado, lo que requiere de un diseño cuidadoso y planificado de las actividades universitarias a realizarse en el medio. Estas actividades que son puntuales, y muchas veces necesarias, pueden agotar a la población con respecto a "recibir" a los/as universitarios/as. Se entiende que el ideal sería la conformación de programas de prácticas integrales de largo plazo en los que puedan participar todos aquellos servicios en sus diferentes funciones. Sin embargo, no se debe desconocer que tanto desde la Universidad como desde la población se demandan actividades puntuales que persiguen resultados inmediatos. La sobrecarga de estas actividades en un espacio geográfico limitado atenta contra la puesta en práctica de la dimensión metodológica de la extensión.

Por último, la escasa descentralización, si bien no es la única dificultad, es un elemento transversal a la implementación de las prácticas de extensión. Quizás el ejemplo más claro sea en las dimensiones ética y política de la extensión universitaria. Si bien la UdelaR se ha encaminado en un profundo proceso de descentralización que tiene como pilares la creación de los Centros Universitarios Regionales, al interior de cada CENUR conviven servicios descentralizados y servicios desconcentrados. Los órganos de cogobierno de los CENUR y de los Centros Universitarios no cuentan aún con líneas políticas claras en cuanto a los planes de estudio, a las políticas de investigación ni de extensión. Las propuestas de trabajo que surgen en el contexto local deben, inevitablemente, negociarse a nivel central, tanto en los servicios como en los órganos centrales de cogobierno. Se vuelve imperioso, de este modo, la generación de políticas locales de enseñanza, investigación y extensión, lo que requiere a su vez una mayor profundización de los niveles de descentralización de los servicios universitarios.
3) Ciclos Iniciales Optativos de Áreas, Por ejemplo, el ClO del Área Social, que permite a sus egresados continuar carreras de Ciencias Sociales, Humanidades, Comunicación, Economía,
Psicología; y el ClO Ciencia y Tecnología, que permite la continuación en carreras de Física, Matemática,

Química, Ingeniería. 


\section{Reflexiones finales}

Descentralizar implica ceder. Cuando se hace referencia a un proceso de descentralización se habla de ceder desde un centro hacia una periferia. Es, por lo tanto, un proceso de redistribución de poder y una reorganización de las correlaciones de fuerza. La implementación de políticas locales de extensión, investigación y enseñanza que confluyen en el diseño de programas integrales necesita, por lo señalado, de una excelencia académica acompañada de un adecuado nivel de recursos (humanos, monetarios, de infraestructura, de transporte, de acceso al conocimiento, entre otros).

Existe aún un largo camino por recorrer a nivel local respecto de la formación y discusión sobre qué modelo de universidad se quiere y cuáles son los mecanismos para alcanzarlo. La extensión universitaria se ofrece como un señuelo hacia el camino de las prácticas integrales; las dimensiones de la extensión universitaria crítica permiten observar nuevos horizontes en la formación universitaria y en su vínculo con la sociedad, al punto de que deberíamos dejar de señalar que son dos espacios distintos. Sin embargo, el proceso de formación y discusión académica, apenas naciente, no es suficiente para la consecución de políticas universitarias locales. La discusión política e institucional tiene un rol fundamental. Los diseños institucionales deben adaptarse a los planes académicos y no a la inversa. Los límites de estructura administrativa, los límites de estructuras políticas, deben transformarse en fronteras, en espacios de encuentro y de negociación, y dejar de ser espacios de contención.

EI PICASA, en este contexto, se presenta como un buen intento, como una buena idea que ha logrado prender en la dura madera, en el viejo árbol. La puesta en práctica de acciones de transformación social desde la Universidad implican transformarla, y ello supone al mismo tiempo transformar a los/as universitarios/as. La implementación del PICASA ha permitido visualizar un camino de transformación y sobre todo identificar la necesaria transformación. Un nuevo modelo de enseñanza universitaria se vuelve impostergable, una pedagogía universitaria emergente que interpele y sea interpelada por la realidad en la realidad junto con un otro que es poseedor de un saber y validado como tal. La concepción de ese otro contempla a todos los actores involucrados en una relación de poder y de saber que parte de la multiplicidad con el objetivo de que se complejice y se diversifique aún más en el encuentro.

\section{Referencias bibliográficas}

Adorno, T. y Horkheimer, M. (1998). Dialéctica de la llustración. Fragmentos filosóficos. Madrid: Trotta.

Bordoli, E. Aportes para pensar la extensión universitaria. In Extensión en Obra. SCEAM.

Carrizo, L. (2010). Interdisciplinariedad y valores. En Toro, B.; Tallone, A., Educación, valores y ciudadanía (pp. 171-184). Madrid: OEl, Fundación SM.

Fals Borda, O. (1981). Investigación participativa y praxis rural (con otros). Lima: Mosca Azul.

Freire, P. (1973). ¿Extensión o comunicación? Rio de Janeiro: Continuum. Freire, P. ([1967] 1989). La educación como práctica de la libertad. Introducción Francis C. Weffort (19 a ed.). Rio de Janeiro: Paz e Terra.

INE (2011). Censo de población y viviendas. Instituto Nacional de Estadística, Uruguay. Disponible en: www.INE.gub.uy

Marx, K. (1966). Tesis sobre Feuerbach. En Marx y Engels, Obras escogidas (pp. 404-406). Moscú: Edit. Progreso.

Montero, M. (2012). El Concepto de Intervención Social desde una Perspectiva Psicológico-Comunitaria. Revista MEC -EDUPAZ, Reserva 04-2011, (l, septiembre-marzo). México: UNAM.

Piedracueva, M. (2016). Discusiones ontológicas sobre una tipología de territorios. NERA, 19 (30), 10-30. Universidade Estadual Paulista.

Tommasino y otros (2010). De la extensión a las prácticas integrales. Hacia la Reforma Universitaria: En la extensión en la renovación de la enseñanza. Espacios de formación integral (10). Montevideo: UdelaR. Disponible en: http://www.extension. edu.uy/system/files_force/Hacia\%20la\%20reforma\%20universitaria\%3A\%20 la\%20extensi\%C3\%B3n\%20en\%20la\%20renovaci\%C3\%B3n\%20de\%20la\%20 ense\%C3\%B1anza.pdf (consultada el 23/5/2016).

Tommasino, H. y Rodríguez, N. (2010). Tres tesis básicas sobre extensión y prácticas integrales en la Universidad de la República. En Arocena, R., Integralidad: tensiones y perspectivas. Cuadernos de Extensión, (1), 19-42. Montevideo: CSEAM-UDELAR.

UdelaR CDC (2009). Para la renovación de la enseñanza y la curricularización de la extensión y las actividades en el medio. Resolución n 5 Consejo Directivo Central de la Universidad de la República. 27 de octubre de 2009. Disponible en: http://www.expe.edu.uy/expe/resoluci.nsf/e3365ff03c2a3d6103256dcc003b9031/ f200247a7f556d23032576550069e060?OpenDocument

UdelaR CCEA. Comisión de Extensión (2008). Extensión: síntesis necesaria y lineamientos de trabajo.

UdelaR CSEAM (2011). Lineamientos generales para el avance de la curricularización de la extensión y generalización de las prácticas integrales en la Universidad de la República. Documento enviado al Consejo Directivo Central en diciembre de 2011. 